 \\ TATRA \\ MOUNTaiNs \\ Mathematical Publications}

DOI: $10.2478 /$ tmmp-2014-0033

Tatra Mt. Math. Publ. 61 (2014), 141-161

\title{
OSCILLATION CRITERIA FOR THIRD ORDER NEUTRAL NONLINEAR DYNAMIC EQUATIONS WITH DISTRIBUTED DEVIATING ARGUMENTS ON TIME SCALES
}

\author{
Taher S. Hassan — Said R. Grace
}

\begin{abstract}
Some new oscillation criteria for third order neutral nonlinear dynamic equations with distributed deviating arguments on time scales are established. The obtained results extend, improve and correlate many known oscillation results for third order dynamic equations.
\end{abstract}

\section{Introduction}

This paper is concerned with the oscillatory behavior of third order neutral nonlinear dynamic equations with distributed deviating arguments on time scales

$$
\left(a(t)\left(\left(x(t)+\int_{c}^{d} p(t, \theta) x(h(t, \theta)) \Delta \theta\right)^{\Delta \Delta}\right)^{\alpha}\right)^{\Delta}+\int_{a}^{b} q(t, \tau) x^{\lambda}(g(t, \tau)) \Delta \tau=0
$$

and

$$
\left(a(t)\left(\left(x^{\beta}\left(h_{1}(t)\right)-r(t) x^{\gamma}\left(h_{2}(t)\right)\right)^{\Delta \Delta}\right)^{\alpha}\right)^{\Delta}+\int_{a}^{b} q(t, \tau) x^{\lambda}(g(t, \tau)) \Delta \tau=0,
$$

on an arbitrary time scale $\mathbb{T} \subseteq \mathbb{R}$ with $\sup \mathbb{T}=\infty, 0<a<b$ and $0<c<d$. We assume that:

(i) $\alpha, \beta, \gamma$ and $\lambda$ are the ratios of positive odd integers;

(C) 2014 Mathematical Institute, Slovak Academy of Sciences.

2010 Mathematics Subject Classification: 34C10, 34C15, 34N05, 34K11, 39A10.

Keywords: oscillation, third order, neutral, dynamic equations, time scales. 
(ii) $a, r: \mathbb{T} \rightarrow[0, \infty), a(t)>0$ are real valued, rd-continuous functions and

$$
\int_{t_{0}}^{\infty} a^{-1 / \alpha}(s) \Delta s=\infty, \quad t_{0} \in \mathbb{T} ;
$$

(iii) $q: \mathbb{T} \times[a, b] \rightarrow[0, \infty)$ and $p: \mathbb{T} \times[c, d] \rightarrow[0, \infty)$ are real valued, rd-continuous functions;

(iv) $g: \mathbb{T} \times[a, b] \rightarrow \mathbb{T}$ and $h: \mathbb{T} \times[c, d] \rightarrow \mathbb{T}$ are nonincreasing with respect to second variable,

$$
g(t, \tau) \leq t \quad \text { and } \quad \lim _{t \rightarrow \infty} g(t, \tau)=\infty, \quad \tau \in[a, b],
$$

and

$$
h(t, \theta) \leq t \quad \text { and } \quad \lim _{t \rightarrow \infty} h(t, \theta)=\infty, \quad \theta \in[c, d] ;
$$

(v) $h_{i}: \mathbb{T} \rightarrow \mathbb{T}$ for $i=1,2$, are real valued, rd-continuous nondecreasing functions such that $h_{i}(t) \leq t$ for $t \geq t_{0} \in \mathbb{T}$ and $\lim _{t \rightarrow \infty} h_{i}(t)=\infty$ for $i=1,2$.

We recall that a solution $x$ of the equation (1.1) (respectively the equation (1.2) is said to be nonoscillatory if there exists $t_{0} \in \mathbb{T}$ such that $x(t) x(\sigma(t))>0$ for all $t \in\left[t_{0}, \infty\right)_{\mathbb{T}}$; otherwise, it is said to be oscillatory. The equation (1.1) (respectively the equation (1.2)) is said to be oscillatory if all its extendible solutions are oscillatory.

Neutral differential equations appear in modelling of the networks containing lossless transmission lines, in the study of vibrating masses attached to an elastic bar, as the Euler equation in some variational problems. In the theory of automatic control and in neuro-mechamial systems in which inertia plays an important role; see [11.

In recent years, there has been much research activity concerning the oscillation theory and applications of dynamic equations, see [1]-[10, [13-[18] and the references contained therein. Particularly, the study content of oscillatory criteria of first and second dynamic equations on time scales is rich. In contrast, the study of oscillation criteria of third order dynamic equations is relatively less. Some interesting results have been obtained concerning the oscillatory and asymptotic behavior of some special cases of the equations (1.1) and (1.2); see [9, [12]. To the best of our knowledge, the oscillatory behavior of (1.1) and (1.2) have not been studied up to now.

The purpose of this paper is to establish some new criteria for the equations (1.1) and (1.2) by using the approach to reduce the problem is such a way that specific oscillation results for first and second order dynamic equations can be 


\section{THIRD ORDER NONLINEAR OSCILLATION}

adapted for the third order case. In Section 2, we investigate the oscillatory behaviour of the equation (1.1) while Section 3 is devoted to study of oscillatory properties of the equation (1.2). The obtained results extend, improve and correlate many of the known oscillation results appeared in the literature that deal with special cases of the equations (1.1) and (1.2).

\section{Oscillation of the equation (1.1)}

In this section we begin with the following lemmas that are essential in the proofs of our results. For simplicity in what follows, whenever we write " $t \geq t_{1}$ " we mean " $t \in\left[t_{1}, \infty\right) \cap \mathbb{T}$ ". It will be convenient to set

$$
y(t):=x(t)+\int_{c}^{d} p(t, \theta) x(h(t, \theta)) \Delta \theta .
$$

Equation (1.1) can be written as

$$
\left(a(t)\left(y^{\Delta \Delta}(t)\right)^{\alpha}\right)^{\Delta}+\int_{a}^{b} q(t, \tau) x^{\lambda}(g(t, \tau)) \Delta \tau=0 .
$$

LEMMA 2.1. Let the condition (1.3) hold and assume that $x$ is an eventually positive solution of the equation (1.1). Then there are only two possible cases for the behaviour of $y$ for large $t \geq t_{0}$ :

(I) $y(t)>0, y^{\Delta}(t)>0, y^{\Delta \Delta}(t)>0$ and $\left(a(t)\left(y^{\Delta \Delta}(t)\right)^{\alpha}\right)^{\Delta} \leq 0$;

(II) $y(t)>0, y^{\Delta}(t)<0, y^{\Delta \Delta}(t)>0$ and $\left(a(t)\left(y^{\Delta \Delta}(t)\right)^{\alpha}\right)^{\Delta} \leq 0$.

LEMMA 2.2. Let condition (1.3) hold and assume that $x$ is an eventually positive solution of equation (1.1) and the corresponding y satisfies Case (I) of Lemma 2.1. If

then

$$
0 \leq P(t):=\int_{c}^{d} p(t, \theta) \Delta \theta \leq p^{*}<1
$$

$$
x(t) \geq\left(1-p^{*}\right) y(t), \quad \text { eventually }
$$

Proof. Since $x$ is an eventually positive solution of the equation (1.1) and the corresponding $y$ is satisfying Case (I) of Lemma 2.1. Then there exists a $t_{1} \geq t_{0} \in \mathbb{T}$ such that

$$
x(t)>0, x(h(t, \theta))>0 \quad \text { and } \quad y^{\Delta}(t)>0 \quad \text { for } \quad t \geq t_{1} \text { and } \theta \in[c, d] .
$$


Now,

$$
\begin{aligned}
x(t) & =y(t)-\int_{c}^{d} p(t, \theta) x(h(t, \theta)) \Delta \theta \\
& \geq y(t)-\int_{c}^{d} p(t, \theta) y(h(t, \theta)) \Delta \theta \\
& \geq y(t)-\left(\int_{c}^{d} p(t, \theta) \Delta \theta\right) y(t) \\
& =\left(1-\int_{c}^{d} p(t, \theta) \Delta \theta\right) y(t) \quad \text { for } \quad t \geq t_{1} .
\end{aligned}
$$

This completes the proof.

LEMMA 2.3. Let the condition (1.3) hold and assume that $x$ is an eventually positive solution of the equation (1.1) and the corresponding y satisfies Case (I) of Lemma 2.1. Then for $t \geq t_{1} \in \mathbb{T}$

$$
y(t) \geq\left(a(t)\left(y^{\Delta \Delta}(t)\right)^{\alpha}\right)^{1 / \alpha} \int_{t_{1}}^{t} \int_{t_{1}}^{u} a^{-1 / \alpha}(s) \Delta s \Delta u .
$$

Proof. Since $x$ is an eventually positive solution of the equation (1.1) and the corresponding $y$ is satisfying Case (I) of Lemma 2.1. Then there exists $t_{1} \geq t_{0} \in \mathbb{T}$ such that

$$
x(t)>0 \quad \text { and } \quad x(h(t, \theta))>0 \quad \text { for } t \geq t_{1} \text { and } \theta \in[c, d] .
$$

By using the fact that $a(t)\left(y^{\Delta \Delta}(t)\right)^{\alpha}$ is decreasing for $t \geq t_{1}$, we have

$$
\begin{aligned}
y^{\Delta}(t) & \geq y^{\Delta}(t)-y^{\Delta}\left(t_{1}\right) \\
& =\int_{t_{1}}^{t}\left(a(s)\left(y^{\Delta \Delta}(s)\right)^{\alpha}\right)^{1 / \alpha} a^{-1 / \alpha}(s) \Delta s \\
& \geq\left(a(t)\left(y^{\Delta \Delta}(t)\right)^{\alpha}\right)^{1 / \alpha} \int_{t_{1}}^{t} a^{-1 / \alpha}(s) \Delta s .
\end{aligned}
$$

Integrating this inequality from $t_{1}$ to $t$, we obtain the desired result. 


\section{THIRD ORDER NONLINEAR OSCILLATION}

Let

$$
Q(t):=\int_{a}^{b} q(t, \tau) \Delta \tau, \quad g_{1}(t):=g(t, a) \quad \text { and } \quad g_{2}(t):=g(t, b) .
$$

In the following result, we employ the following auxiliary equation

$$
z^{\Delta}(t)+\left(1-p^{*}\right)^{\lambda} Q(t)\left(\int_{t_{1}}^{g_{2}(t)} \int_{t_{1}}^{u} a^{-1 / \alpha}(s) \Delta s \Delta u\right)^{\lambda} z^{\lambda / \alpha}\left(g_{2}(t)\right)=0
$$

for $t \geq t_{1}, t_{1} \in\left[t_{0}, \infty\right)_{\mathbb{T}}$.

LEMMA 2.4. Let the conditions (1.3) and (2.3) hold and the equation (2.7) be oscillatory, then the equation (1.1) has no eventually positive solution $x$ such that $y$ defined by (2.1) satisfies Case (I) of Lemma 2.1.

P r o of. Let $t_{0} \in \mathbb{T}$ be sufficiently large such that $x(t)>0, x(h(t, \theta))>0$ and $x(g(t, \tau))>0$ for $t \geq t_{0}, \theta \in[c, d]$ and $\tau \in[a, b]$ and assume that $y$ satisfies Case (I) of Lemma 2.1. Using (2.4) and (2.5) in the equation (2.2), we get

$$
z^{\Delta}(t)+\left(1-p^{*}\right)^{\lambda} Q(t)\left(\int_{t_{1}}^{g_{2}(t)} \int_{t_{1}}^{u} a^{-1 / \alpha}(s) \Delta s \Delta u\right)^{\lambda} z^{\lambda / \alpha}\left(g_{2}(t)\right) \leq 0,
$$

for $t_{1} \in\left[t_{0}, \infty\right)_{\mathbb{T}}$, where $z(t):=a(t)\left(y^{\Delta \Delta}(t)\right)^{\alpha}>0$. Integrating (2.8) from $t$ to $u \geq t$ and letting $u \rightarrow \infty$, we have

$$
z(t) \geq G(t, z(t)),
$$

where

$$
G(t, z(t)):=\left(1-p^{*}\right)^{\lambda} \int_{t}^{\infty} Q(v)\left(\int_{t_{1}}^{g_{2}(v)} \int_{t_{1}}^{u} a^{-1 / \alpha}(s) \Delta s \Delta u\right)^{\lambda} z^{\lambda / \alpha}\left(g_{2}(v)\right) \Delta v .
$$

Now, we define a sequence of successive approximations $\left\{w_{j}(t)\right\}$ as follows:

$$
\begin{aligned}
w_{0}(t) & :=z(t), \\
w_{j+1}(t) & :=G\left(t, w_{j}(t)\right), \quad j=0,1,2, \ldots .
\end{aligned}
$$

It is easy to show that

$$
0<w_{j}(t) \leq z(t) \quad \text { and } \quad w_{j+1}(t) \leq w_{j}(t), \quad j=0,1,2, \ldots
$$

Then, the sequence $\left\{w_{j}(t)\right\}$ is nonincreasing and bounded for each $t \geq t_{1}$. This means that we may define $w(t):=\lim _{j \rightarrow \infty} w_{j}(t) \geq 0$. Since

$$
0 \leq w(t) \leq w_{j}(t) \leq z(t) \quad \text { for all } \quad j \geq 0 .
$$


By the Lebesgue's dominated convergence theorem on time scale, one can easily find

Therefore,

$$
w(t)=G(t, w(t))
$$

$$
w^{\Delta}(t)=-\left(1-p^{*}\right)^{\lambda} Q(t)\left(\int_{t_{1}}^{g_{2}(t)} \int_{t_{1}}^{u} a^{-1 / \alpha}(s) \Delta s \Delta u\right)^{\lambda} w^{\lambda / \alpha}\left(g_{2}(t)\right) .
$$

Hence the equation (2.7) has a positive solution $w(t)$. This completes the proof.

In the case when $\lambda=\alpha$ in the inequality (2.8), we state the following lemma.

LEMma 2.5 ([1]). If $\lambda=\alpha$,

$$
\limsup _{t \rightarrow \infty} \sup _{\xi \in E}\left\{\xi e_{-\xi \eta}\left(t, g_{2}(t)\right)\right\}<1
$$

where

$$
E:=\{\xi: \xi>0,1-\xi \eta(t) \mu(t)>0\}
$$

and

$$
\eta(t):=\left(1-p^{*}\right)^{\alpha} Q(t)\left(\int_{t_{1}}^{g_{2}(t)} \int_{t_{1}}^{u} a^{-1 / \alpha}(s) \Delta s \Delta u\right)^{\alpha}
$$

then the inequality (2.8) has no eventually positive solution.

LEMMA 2.6. Let the condition (1.3) hold and assume that $x(t)$ is an eventually positive solution the equation (11.1) and the corresponding y satisfies Case (I) of Lemma 2.1. Then there exists $t_{1} \in\left[t_{0}, \infty\right)_{\mathbb{T}}$ such that

$$
y(t) \geq y^{\Delta}(t) A\left(t, t_{1}\right) \quad \text { for } \quad t \in\left(t_{1}, \infty\right)_{\mathbb{T}},
$$

where

$$
A\left(t, t_{1}\right):=\frac{\int_{t_{1}}^{t} \int_{t_{1}}^{u} a^{-1 / \alpha}(s) \Delta s \Delta u}{\int_{t_{1}}^{t} a^{-1 / \alpha}(s) \Delta s} .
$$

P r o of. Let $t_{0} \in \mathbb{T}$ be sufficiently large such that $x(t)>0, x(h(t, \theta))>0$ and $x(g(t, \tau))>0$ for $t \geq t_{0}, \theta \in[c, d]$ and $\tau \in[a, b]$ and assume that $y$ satisfies Case (I) of Lemma 2.1 for $t \geq t_{0}$. From Lemma 2.3, we have

$$
y^{\Delta}(t) \geq\left(a(t)\left(y^{\Delta \Delta}(t)\right)^{\alpha}\right)^{1 / \alpha} \int_{t_{1}}^{t} a^{-1 / \alpha}(s) \Delta s \quad \text { for } \quad t \geq t_{1} \geq t_{0} .
$$

Note that

$$
\left[\frac{y^{\Delta}(t)}{\int_{t_{1}}^{t} a^{-1 / \alpha}(s) \Delta s}\right]^{\Delta}=\frac{a^{-1 / \alpha}(t)\left[\left(a(t)\left(y^{\Delta \Delta}(t)\right)^{\alpha}\right)^{1 / \alpha} \int_{t_{1}}^{t} a^{-1 / \alpha}(s) \Delta s-y^{\Delta}(t)\right]}{\int_{t_{1}}^{t} a^{-1 / \alpha}(s) \Delta s \int_{t_{1}}^{\sigma(t)} a^{-1 / \alpha}(s) \Delta s},
$$




\section{THIRD ORDER NONLINEAR OSCILLATION}

we have

$$
\left[\frac{y^{\Delta}(t)}{\int_{t_{1}}^{t} a^{-1 / \alpha}(s) \Delta s}\right]^{\Delta}<0 \quad \text { for } \quad t \in\left(t_{1}, \infty\right)_{\mathbb{T}} .
$$

Then

$$
\begin{aligned}
y(t) & \geq y(t)-y\left(t_{1}\right) \\
& =\int_{t_{1}}^{t} \frac{y^{\Delta}(u)}{\int_{t_{1}}^{u} a^{-1 / \alpha}(s) \Delta s}\left(\int_{t_{1}}^{u} a^{-1 / \alpha}(s) \Delta s\right) \Delta u \\
& \geq \frac{y^{\Delta}(t)}{\int_{t_{1}}^{t} a^{-1 / \alpha}(s) \Delta s} \int_{t_{1} t_{1}}^{t} a^{-1 / \alpha}(s) \Delta s \Delta u \\
& =y^{\Delta}(t) A\left(t, t_{1}\right) .
\end{aligned}
$$

This completes the proof.

For $g_{2}(t)>t_{0}$, we set

$$
\bar{Q}(t):=\left(1-p^{*}\right)^{\lambda} A^{\lambda}\left(g_{2}(t), t_{0}\right) Q(t) .
$$

The hypotheses of next two lemmas include knowledge of the behaviour of the solution of the second order dynamic equation

$$
\left(a(t)\left(z^{\Delta}(t)\right)^{\alpha}\right)^{\Delta}+\bar{Q}(t) z^{\lambda}\left(g_{2}(t)\right)=0,
$$

LEMMA 2.7. If the conditions (1.3) and (2.3) hold and the equation (2.10) is oscillatory, then the equation (1.1) has no eventually positive solution $x$ such that $y$ is defined by (2.1) which satisfies Case (I) of Lemma 2.1.

P r o of. Let $x(t)$ be an eventually positive solution of the equation (1.1), say $x(t)>0$ and $x(h(t, \theta))>0$ and $x(g(t, \tau))>0$ for $t \geq t_{1}$ for some $t_{1} \in\left[t_{0}, \infty\right)_{\mathbb{T}}$, $\theta \in[c, d]$ and $\tau \in[a, b]$ and assume that $y$ satisfies Case (I) of Lemma 2.1. From (2.9), there exist a constant $k_{1}, 0<k_{1}<1$ and a $t_{2}>t_{1}$ such that

$$
y(t) \geq A\left(t, t_{1}\right) y^{\Delta}(t) \quad \text { for } \quad t \geq t_{2} .
$$

From (2.4), we see that

$$
x(t) \geq\left(1-p^{*}\right) y(t) \quad \text { for } \quad t \geq t_{3},
$$

for some $t_{3} \geq t_{2}$. Hence, there exists $t_{4} \geq t_{3}$ such that

$$
y\left(g_{2}(t)\right) \geq A\left(g_{2}(t), t_{1}\right) y^{\Delta}\left(g_{2}(t)\right) \quad \text { for } \quad t \geq t_{4} .
$$

Using (2.12) and (2.13) in the equation (2.2) we have

$$
\left(a(t)\left(z^{\Delta}(t)\right)^{\alpha}\right)^{\Delta}+\bar{Q}(t) z^{\lambda}\left(g_{2}(t)\right) \leq 0,
$$


for $t \geq t_{4}$, where $z(t):=y^{\Delta}(t)>0$. Integrating (2.14) from $t$ to $u \geq t \geq t_{4}$ and letting $u \rightarrow \infty$, we obtain

$$
z^{\Delta}(t) \geq\left(\frac{1}{a(t)} \int_{t}^{\infty} \bar{Q}(s) z^{\lambda}\left(g_{2}(s)\right) \Delta s\right)^{1 / \alpha} .
$$

Integrating (2.15) from $t_{4}$ to $t \geq t_{4}$, we obtain

$$
z(t) \geq z\left(t_{4}\right)+\int_{t_{4}}^{t}\left(\frac{1}{a(u)} \int_{u}^{\infty} \bar{Q}(s) z^{\lambda}\left(g_{2}(s)\right) \Delta s\right)^{1 / \alpha} \Delta u .
$$

Next, we define a sequence $\left\{w_{m}(t)\right\}_{m \in \mathbb{N}_{0}}$ by

$$
\begin{aligned}
w_{0}(t) & =z(t), \\
w_{m+1}(t) & =z\left(t_{4}\right)+\int_{t_{4}}^{t}\left(\frac{1}{a(u)} \int_{u}^{\infty} \bar{Q}(s) z^{\lambda}\left(g_{2}(s)\right) \Delta s\right)^{1 / \alpha} \Delta u, \quad m \in \mathbb{N}_{0} .
\end{aligned}
$$

It is easy to check by induction that $\left\{w_{m}(t)\right\}$ is a well-defined decreasing sequence satisfying

$$
z\left(t_{4}\right) \leq w_{m}(t) \leq z(t) \quad \text { for } \quad t \geq t_{4} \text { and } m \in \mathbb{N}_{0} .
$$

Thus, there exists a function $w$ on $\left[t_{4}, \infty\right)_{\mathbb{T}}$ such that

$$
\lim _{m \rightarrow \infty} w_{m}(t)=w(t) \quad \text { and } \quad z\left(t_{4}\right) \leq w(t) \leq z(t) .
$$

By the Lebesgue's dominated convergence theorem on time scale, it follows that

$$
w(t)=z\left(t_{4}\right)+\int_{t_{4}}^{t}\left(\frac{1}{a(u)} \int_{u}^{\infty} \bar{Q}(s) w^{\lambda}\left(g_{2}(s)\right) \Delta s\right)^{1 / \alpha} \Delta u .
$$

Differentiating (2.16) twice, we conclude that $w$ is a nonoscillatory solution of the equation (2.10) with the desired property. This completes the proof of the lemma.

The following lemma makes use of the auxiliary equation

$$
\left(\frac{1}{\bar{A}^{\alpha}(t)}\left(v^{\Delta}(t)\right)^{\alpha}\right)^{\Delta}+\left(1-p^{*}\right)^{\lambda} Q(t) v^{\lambda}\left(g_{2}(t)\right)=0 \quad \text { for } \quad t \geq t_{2},
$$

where $\bar{A}(t):=\int_{t_{1}}^{t} a^{-1 / \alpha}(s) \Delta s$ for sufficiently large $t_{2} \in\left(t_{1}, \infty\right)_{\mathbb{T}}$ for some $t_{1} \in\left[t_{0}, \infty\right)_{\mathbb{T}}$.

Lemma 2.8. If the conditions (1.3) and (2.3) hold and the equation (2.17) is oscillatory, then the conclusion of Lemma 2.7 holds. 


\section{THIRD ORDER NONLINEAR OSCILLATION}

P r o of. Let $x(t)$ be an eventually positive solution of the equation (1.1), say $x(t)>0$ and $x(h(t, \theta))>0$ and $x(g(t, \tau))>0$ for $t \geq t_{1}$ for some $t_{1} \in\left[t_{0}, \infty\right)_{\mathbb{T}}$, $\theta \in[c, d]$ and $\tau \in[a, b]$ and let $y$ satisfy Case (I) of Lemma 2.1. Hence

$$
\begin{aligned}
y^{\Delta}(t) & =y^{\Delta}\left(t_{1}\right)+\int_{t_{1}}^{t} \frac{a^{1 / \alpha}(s) y^{\Delta \Delta}(s)}{a^{1 / \alpha}(s)} \Delta s \geq a^{1 / \alpha}(t) y^{\Delta \Delta}(t) \int_{t_{1}}^{t} \frac{\Delta s}{a^{1 / \alpha}(s)} \\
& =a^{1 / \alpha}(t) y^{\Delta \Delta}(t) \bar{A}(t) \quad \text { for } \quad t \geq t_{1} .
\end{aligned}
$$

An integration yields

$$
y(t) \geq y\left(t_{1}\right)+\int_{t_{1}}^{t} \bar{A}(u) a^{1 / \alpha}(u) y^{\Delta \Delta}(u) \Delta u .
$$

Using (2.4) in the equation (2.2), integrating from $u$ to $v \geq u \geq t_{1}$ and letting $v \rightarrow \infty$, we have

$$
y^{\Delta \Delta}(u) \geq \frac{1}{a^{1 / \alpha}(u)}\left(\int_{u}^{\infty}\left(1-p^{*}\right)^{\lambda} Q(s) y^{\lambda}\left(g_{2}(s)\right) \Delta s\right)^{1 / \alpha} .
$$

Substituting (2.19) into (2.18) gives

$$
y(t) \geq y\left(t_{2}\right)+\int_{t_{1}}^{t} \bar{A}(u)\left(\int_{u}^{\infty}\left(1-p^{*}\right)^{\lambda} Q(s) y^{\lambda}\left(g_{2}(s)\right) \Delta s\right)^{1 / \alpha} \Delta u
$$

Next we define the sequences $\left\{v_{m}(t)\right\}_{m \in \mathbb{N}_{0}}$ by

$$
\begin{aligned}
v_{0}(t) & =y(t), \\
v_{m+1}(t) & =y\left(t_{1}\right)+\int_{t_{1}}^{t} \bar{A}(u)\left(\int_{u}^{\infty}\left(1-p^{*}\right)^{\lambda} Q(s) y^{\lambda}\left(g_{2}(s)\right) \Delta s\right)^{1 / \alpha} \Delta u, \quad m \in \mathbb{N}_{0} .
\end{aligned}
$$

The remainder of the proof is similar to the proof of Lemma 2.7 and is omitted.

Next, we present the following result.

LEMmA 2.9. Let the conditions (1.3) and (2.3) hold and assume that $x$ is an eventually positive solution of the equation (1.1) and the corresponding $y$ satisfies Case (II) of Lemma 2.1. Then either

$$
x(t) \geq\left(\frac{1-p^{*} \delta}{\delta}\right) y(t)
$$

eventually, where $\delta>1$ is any constant with $p^{*} \delta<1$ and $p^{*}$ is as in (2.3), or $\lim _{t \rightarrow \infty} x(t)=0$. 
P r o of. Pick $t_{0} \in \mathbb{T}$ such that

$$
x(t)>0 \text { and } x(h(t, \theta))>0 \text { for } t \geq t_{0} \text { and } \theta \in[c, d] .
$$

Also, since $y(t)$ satisfies Case (II) of Lemma 2.1, then there exists a constant $k$ such that

$$
\lim _{t \rightarrow \infty} y(t)=k<\infty \text {. }
$$

(i) Assume that $k>0$, then we have

Now,

$$
k<y(t)<k \delta, \quad \text { for all } \delta>1 \text { and } t \geq t_{0} .
$$

and so

$$
x(t)=y(t)-\int_{c}^{d} p(t, \theta) x(h(t, \theta)) \Delta \theta,
$$

$$
x(t) \geq k-k p^{*} \delta=\left(\frac{1-p^{*} \delta}{\delta}\right) k \delta \geq\left(\frac{1-p^{*} \delta}{\delta}\right) y(t) \quad \text { for } \quad t \geq t_{0} .
$$

(ii) Assume that $k=0$, then $\lim _{t \rightarrow \infty} y(t)=0$. Since $0<x(t) \leq y(t)$ on $\left[t_{0}, \infty\right)_{\mathbb{T}}$, then $\lim _{t \rightarrow \infty} x(t)=0$. This completes the proof of the lemma.

Lemma 2.10. Let the conditions (1.3) and (2.3) hold and assume that $x(t)$ is an eventually positive solution of the equation (1.1) and the corresponding $y$ satisfies Case (II) of Lemma 2.1. If

$$
\int_{t_{0}}^{\infty} \int_{v}^{\infty}\left[\frac{1}{a(u)} \int_{u}^{\infty} Q(s) \Delta s\right]^{1 / \alpha} \Delta u \Delta v=\infty,
$$

then $\lim _{t \rightarrow \infty} x(t)=0$.

P r o of. Proceeding as in the proof of Lemma 2.9, we obtain either (2.20) holds or $\lim _{t \rightarrow \infty} x(t)=0$. We suppose that

$$
x(t) \geq\left(\frac{1-p^{*} \delta}{\delta}\right) y(t), \quad \text { for } \quad t \geq t_{1} \geq t_{0} .
$$

Using (2.23) in the equation (2.2), we have

$$
\begin{aligned}
\left(a(t)\left(y^{\Delta \Delta}(t)\right)^{\alpha}\right)^{\Delta} & =-\int_{a}^{b} q(t, \tau) x^{\lambda}(g(t, \tau)) \Delta \tau \\
& \leq-\left(\frac{1-p^{*} \delta}{\delta}\right)^{\lambda} \int_{a}^{b} q(t, \tau) y^{\lambda}(g(t, \tau)) \Delta \tau \\
& \leq-\left(\frac{1-p^{*} \delta}{\delta}\right)^{\lambda} Q(t) y^{\lambda}\left(g_{1}(t)\right) \quad \text { for } \quad t \geq t_{1} \geq t_{0} .
\end{aligned}
$$




\section{THIRD ORDER NONLINEAR OSCILLATION}

Integrating this inequality from $t$ to $u \geq t \geq t_{1}$ and letting $u \rightarrow \infty$, we have

$$
y^{\Delta \Delta}(t) \geq\left(\frac{1-p^{*} \delta}{\delta}\right)^{\lambda / \alpha}\left(\frac{1}{a(t)} \int_{t}^{\infty} Q(s) y^{\lambda}\left(g_{1}(s)\right) \Delta s\right)^{1 / \alpha} .
$$

Using (2.21) in (2.25), we get

$$
y^{\Delta \Delta}(t) \geq c\left(\frac{1}{a(t)} \int_{t}^{\infty} Q(s) \Delta s\right)^{1 / \alpha}
$$

where $c:=\left(k\left[\frac{1-p^{*} \delta}{\delta}\right]\right)^{\lambda / \alpha}$. Integrating (2.26) twice, we obtain

$$
\infty>y\left(t_{1}\right) \geq c \int_{t_{1}}^{t} \int_{v}^{\infty}\left(\frac{1}{a(u)} \int_{u}^{\infty} Q(s) \Delta s\right)^{1 / \alpha} \Delta u \Delta v \rightarrow \infty \quad \text { as } t \rightarrow \infty,
$$

which is a contradiction. This completes the proof of the lemma.

LEMMA 2.11. Let $g_{1}$ be a nondecreasing on $\left[t_{0}, \infty\right)_{\mathbb{T}}$, conditions (1.3) and (2.3) hold and assume that $x(t)$ is an eventually positive solution of the equation (1.1) and the corresponding y satisfies Case (II) of Lemma 2.1. If

$$
\limsup _{t \rightarrow \infty} \int_{g_{1}(t)}^{t}\left(\frac{1}{a(u)} \int_{u}^{t} Q(s)\left[g_{1}(t)-g_{1}(s)\right]^{\lambda} \Delta s\right)^{1 / \alpha} \Delta u> \begin{cases}c & \text { if } \lambda=\alpha, \\ 0 & \text { if } \lambda<\alpha,\end{cases}
$$

where $Q$ and $g_{1}$ are as in (2.6), $c:=\frac{\delta}{1-p^{*} \delta}, p^{*}$ and $\delta$ are as in Lemma 2.9, then $\lim _{t \rightarrow \infty} x(t)=0$.

P r o o f. Proceeding as in the proof of Lemma 2.10, we obtain (2.24). We also have

$$
\begin{aligned}
-y\left(g_{1}(s)\right) & \leq y\left(g_{1}(t)\right)-y\left(g_{1}(s)\right)=\int_{g_{1}(s)}^{g_{1}(t)} y^{\Delta}(\tau) \Delta \tau \\
& \leq y^{\Delta}\left(g_{1}(t)\right) \int_{g_{1}(s)}^{g_{1}(t)} \Delta \tau=y^{\Delta}\left(g_{1}(t)\right)\left[g_{1}(t)-g_{1}(s)\right],
\end{aligned}
$$

for $t \geq s \geq t_{0}$. Integrating (2.24) from $u$ to $t \geq u \geq t_{0}$, we obtain

$$
y^{\Delta \Delta}(u) \geq\left(\frac{1-p^{*} \delta}{\delta}\right)^{\lambda / \alpha}\left(\frac{1}{a(u)} \int_{u}^{t} Q(s) y^{\lambda}\left(g_{1}(s)\right) \Delta s\right)^{1 / \alpha} .
$$


Substituting (2.28) into (2.29), we obtain

$$
y^{\Delta \Delta}(u) \geq\left(\frac{1-p^{*} \delta}{\delta}\right)^{\lambda / \alpha}\left(\frac{1}{a(u)} \int_{u}^{t} Q(s)\left[g_{1}(t)-g_{1}(s)\right]^{\lambda} \Delta s\right)^{1 / \alpha}\left(-y^{\Delta}\left(g_{1}(t)\right)\right)^{\lambda / \alpha} .
$$

Integrating from $g_{1}(t) \geq t_{0}$ to $t$ gives

$$
\begin{aligned}
& -y^{\Delta}\left(g_{1}(t)\right) \geq y^{\Delta}(t)-y^{\Delta}\left(g_{1}(t)\right) \geq \\
& \left(\frac{1-p^{*} \delta}{\delta}\right)^{\lambda / \alpha}\left(-y^{\Delta}\left(g_{1}(t)\right)\right)^{\lambda / \alpha} \int_{g_{1}(t)}^{t}\left(\frac{1}{a(u)} \int_{u}^{t} Q(s)\left[g_{1}(t)-g_{1}(s)\right]^{\lambda} \Delta s\right)^{1 / \alpha} \Delta u .
\end{aligned}
$$

So

$$
\begin{gathered}
\left(-y^{\Delta}\left(g_{1}(t)\right)\right)^{1-\lambda / \alpha} \geq \\
\left(\frac{1-p^{*} \delta}{\delta}\right)^{\lambda / \alpha} \int_{g_{1}(t)}^{t}\left(\frac{1}{a(u)} \int_{u}^{t} Q(s)\left[g_{1}(t)-g_{1}(s)\right]^{\lambda} \Delta s\right)^{1 / \alpha} \Delta u .
\end{gathered}
$$

Taking limsup as $t \rightarrow \infty$ of both sides of the above inequality. If $\lambda=\alpha$, the contradiction is obvious. If $\lambda<\alpha$, then the left hand side of (2.30) is positive and must decrease to zero (to prevent a contradiction to the positivity of $y(t)$ ). This contradicts (2.27) and completes the proof of the lemma.

LEMMA 2.12. Let the hypotheses of Lemma 2.11 hold with the condition (2.27) be replaced by

$$
\limsup _{t \rightarrow \infty} \int_{g_{1}(t)}^{t} \int_{v}^{t}\left(\frac{1}{a(u)} \int_{u}^{t} Q(s) \Delta s\right)^{1 / \alpha} \Delta u \Delta v> \begin{cases}c & \text { if } \lambda=\alpha \\ 0 & \text { if } \lambda<\alpha .\end{cases}
$$

Then the conclusion of Lemma 2.11 holds.

P r o o f. As in the proof of Lemma 2.11, we obtain (2.29) and integrating we have

$$
\begin{aligned}
-y^{\Delta}(v) & \geq y^{\Delta}(t)-y^{\Delta}(v) \\
& \geq\left(\frac{1-p^{*} \delta}{\delta}\right)^{\lambda / \alpha} y^{\lambda / \alpha}\left(g_{1}(t)\right) \int_{v}^{t}\left(\frac{1}{a(u)} \int_{u}^{t} Q(s) \Delta s\right)^{1 / \alpha} \Delta u .
\end{aligned}
$$

Integrating from $g_{1}(t) \geq t_{0}$ to $t$ yields

$$
y^{1-\lambda / \alpha}\left(g_{1}(t)\right) \geq\left(\frac{1-p^{*} \delta}{\delta}\right)^{\lambda / \alpha} \int_{g_{1}(t)}^{t} \int_{v}^{t}\left(\frac{1}{a(u)} \int_{u}^{t} Q(s) \Delta s\right)^{1 / \alpha} \Delta u \Delta v .
$$




\section{THIRD ORDER NONLINEAR OSCILLATION}

Taking limsup as $t \rightarrow \infty$ gives a contradiction to the condition (2.31). This completes the proof of the lemma.

We are now ready to present the main results in this section.

Theorem 2.1. Let (1.3), (2.3), (2.22) and either one of the equations (2.7), (2.10) or (2.17) be oscillatory, then every solution $x(t)$ of the equation (1.1) oscillates or satisfies $\lim _{t \rightarrow \infty} x(t)=0$.

P r o of. Let $x(t)$ be an eventually positive solution of the equation (1.1), say

$$
x(t)>0 \quad \text { and } \quad x(h(t, \theta))>0 \text { and } x(g(t, \tau))>0
$$

for $t \geq t_{1}$ for some $t_{1} \in\left[t_{0}, \infty\right)_{\mathbb{T}}, \theta \in[c, d]$ and $\tau \in[a, b]$. Then $y(t)$ satisfies one of the two cases of Lemma 2.1. By either Lemmas 2.4, 2.7 or 2.8, Case (I) cannot hold. If Case (II) holds, Lemma 2.10 implies $\lim _{t \rightarrow \infty} x(t)=0$. This proves the theorem.

Next, we establish another new oscillation criteria for the equation (1.1).

TheOREM 2.2. Let $\lambda \leq \alpha, g_{1}$ be a nondecreasing on $\left[t_{0}, \infty\right)_{\mathbb{T}}$, (1.3) and (2.3) hold. If either one of the equations (2.7), (2.10) or (2.17) is oscillatory, and condition (2.27) or (2.31) holds, then every solution $x(t)$ of the equation (1.1) oscillates or satisfies $\lim _{t \rightarrow \infty} x(t)=0$.

Pr o of. Let $x(t)$ be an eventually positive solution of the equation (1.1), say

$$
x(t)>0 \quad \text { and } \quad x(h(t, \theta))>0 \quad \text { and } \quad x(g(t, \tau))>0
$$

for $t \geq t_{1}$ for some $t_{1} \in\left[t_{0}, \infty\right)_{\mathbb{T}}, \theta \in[c, d]$ and $\tau \in[a, b]$. Then $y(t)$ satisfies one of the two cases of Lemma 2.1. By either Lemmas 2.4, 2.7 or 2.8. Case (I) cannot hold. By Lemma 2.11 (or Lemma 2.12), Case (II) does not hold. This completes the proof of the theorem.

Remark 1. Our results of this section remain valid of $g(t, \tau)$ is nondecreasing in the second variable. In this case, we replace

and

$$
g_{1}(t) \quad \text { by } \quad \bar{g}_{1}(t)=g(t, b)
$$

$$
g_{2}(t) \quad \text { by } \quad \bar{g}_{2}(t)=g(t, a) .
$$

Remark 2. We may apply Lemma 2.5 to equation (2.7) with $\lambda=\alpha$. This details are left to the readers. 


\section{Oscillation of the equation (1.2)}

We begin with the following lemmas that are essential in the proof of our theorems. It will be convenient to set

$$
y(t)=x^{\beta}\left(h_{1}(t)\right)-r(t) x^{\gamma}\left(h_{2}(t)\right) .
$$

The equation (1.2) can then be written as

$$
\left(a(t)\left(y^{\Delta \Delta}(t)\right)^{\alpha}\right)^{\Delta}+\int_{a}^{b} q(t, \tau) x^{\lambda}(g(t, \tau)) \Delta \tau=0 .
$$

LEMMA 3.1. Let the condition (1.3) hold and assume that $x$ is an eventually positive solution of the equation (1.2). Then there are only three possible cases for the behaviour of $y$ for large $t \geq t_{0}$ :

(I) $y(t)>0, y^{\Delta}(t)>0, y^{\Delta \Delta}(t)>0$, and $\left(a(t)\left(y^{\Delta \Delta}(t)\right)^{\alpha}\right)^{\Delta} \leq 0$;

(II) $y(t)>0, y^{\Delta}(t)<0, y^{\Delta \Delta}(t)>0$, and $\left(a(t)\left(y^{\Delta \Delta}(t)\right)^{\alpha}\right)^{\Delta} \leq 0$;

(III) $y(t)<0, y^{\Delta}(t)<0, y^{\Delta \Delta}(t)>0$, and $\left(a(t)\left(y^{\Delta \Delta}(t)\right)^{\alpha}\right)^{\Delta} \leq 0$.

If case (I) or (II) holds, then we find

$$
x(t) \geq y^{1 / \beta}\left(h_{1}^{-1}(t)\right) .
$$

Using (3.3) in the equation (3.2), we have

$$
\left(a(t)\left(y^{\Delta \Delta}(t)\right)^{\alpha}\right)^{\Delta}+\int_{a}^{b} q(t, \tau) y^{\lambda / \beta}\left(h_{1}^{-1} \circ g(t, \tau)\right) \Delta \tau \leq 0,
$$

and when Case (III) holds, we see that

$$
0<z(t)=-y(t)=r(t) x^{\gamma}\left(h_{2}(t)\right)-x^{\beta}\left(h_{1}(t)\right) \leq r(t) x^{\gamma}\left(h_{2}(t)\right),
$$

and so

$$
x(t) \geq\left(\frac{z\left(h_{2}^{-1}(t)\right)}{r\left(h_{2}^{-1}(t)\right)}\right)^{1 / \gamma}
$$

and the equation (3.2) becomes

$$
\left(a(t)\left(z^{\Delta \Delta}(t)\right)^{\alpha}\right)^{\Delta} \geq \int_{a}^{b} q(t, \tau) r^{-\lambda / \gamma}\left(h_{2}^{-1} \circ g(t, \tau)\right) z^{\lambda / \gamma}\left(h_{2}^{-1} \circ g(t, \tau)\right) \Delta \tau .
$$

Next, we assume that

(vi) $\zeta_{1}(t, \tau)=h_{1}^{-1} \circ g(t, \tau) \leq t$ for $\tau \in[a, b], \zeta_{1}$ is nonincreasing with respect to the second variable and $\lim _{t \rightarrow \infty} \zeta_{1}(t, \tau)=\infty$; 


\section{THIRD ORDER NONLINEAR OSCILLATION}

(vii) $\zeta_{2}(t, \tau)=h_{2}^{-1} \circ g(t, \tau) \leq t$ for $\tau \in[a, b], \zeta_{2}$ is nonincreasing with respect to the second variable and $\lim _{t \rightarrow \infty} \zeta_{2}(t, \tau)=\infty$.

Also, we set

$$
Q(t):=\int_{a}^{b} q(t, \tau) \Delta \tau, \hat{\zeta}_{1}(t):=\zeta_{1}(t, a), \bar{\zeta}_{1}(t):=\zeta_{1}(t, b),
$$

and

$$
\hat{\zeta}_{2}(t):=\zeta_{2}(t, a), \bar{\zeta}_{2}(t):=\zeta_{2}(t, b)
$$

Now, if $y$ satisfies Case (I) of Lemma 3.1, then (3.4) becomes

$$
\left(a(t)\left(y^{\Delta \Delta}(t)\right)^{\alpha}\right)^{\Delta}+Q(t) y^{\lambda / \beta}\left(\bar{\zeta}_{1}(t)\right) \leq 0
$$

where $Q$ is as in (2.6). When $y$ satisfies Case (II) of Lemma 3.1, then (3.4) becomes

$$
\left(a(t)\left(y^{\Delta \Delta}(t)\right)^{\alpha}\right)^{\Delta}+Q(t) y^{\lambda / \beta}\left(\hat{\zeta}_{1}(t)\right) \leq 0 .
$$

If $y$ satisfies Case (III) of Lemma 3.1, then (3.6) takes the form

$$
\left(a(t)\left(z^{\Delta \Delta}(t)\right)^{\alpha}\right)^{\Delta} \geq \hat{Q}(t) z^{\lambda / \gamma}\left(\bar{\zeta}_{2}(t)\right)
$$

where

$$
\hat{Q}(t):=\int_{a}^{b} q(t, \tau) r^{-\lambda / \gamma}\left(\zeta_{2}(t, \tau)\right) \Delta \tau
$$

As direct consequence of Lemmas 2.4, 2.6, 2.7, 2.8, 2.10, 2.11 and 2.12, we get the following results.

LEMMA 3.2. Let the condition (1.3) hold and equation

$$
z^{\Delta}(t)+Q(t)\left(\int_{t_{1}}^{\bar{\zeta}_{1}(t)} \int_{t_{1}}^{u} a^{-1 / \alpha}(s) \Delta s \Delta u\right)^{\lambda / \beta} z^{\lambda /(\alpha \beta)}\left(g_{2}(t)\right)=0 \quad \text { for } t \geq t_{1},
$$

for $t_{1} \in\left[t_{0}, \infty\right)_{\mathbb{T}}$, is oscillatory, then the equation (1.2) has no eventually positive solution $x$ such that $y$ defined by (3.1) satisfies Case (I) of Lemma 3.1.

LEMMA 3.3. Let the condition (1.3) hold and assume that $x(t)$ is an eventually positive solution of the equation (1.2) and the corresponding y satisfies Case (I) of Lemma 3.1. Then there exists $t_{1} \in\left[t_{0}, \infty\right)_{\mathbb{T}}$ such that (2.9) holds.

LEMMA 3.4. If the condition (1.3) holds and the equation

$$
\left(a(t)\left(z^{\Delta}(t)\right)^{\alpha}\right)^{\Delta}+\bar{Q}(t) z^{\lambda}\left(g_{2}(t)\right)=0
$$

where for $\bar{\zeta}_{1}(t)>t_{0}$, we set

$$
\bar{Q}(t):=A^{\lambda}\left(\bar{\zeta}_{1}(t), t_{0}\right) Q(t)
$$


is oscillatory, then the equation (1.2) has no eventually positive solution $x$ such that $y$ is defined by (3.1) satisfies Case (I) of Lemma 3.1.

LEMMA 3.5. If the condition (1.3) hold and the equation

$$
\left(\frac{1}{\bar{A}^{\alpha}(t)}\left(v^{\Delta}(t)\right)^{\alpha}\right)^{\Delta}+Q(t) v^{\lambda}\left(g_{2}(t)\right)=0 \quad \text { for } \quad t \geq t_{2},
$$

where $\bar{A}(t):=\int_{t_{1}}^{t} a^{-1 / \alpha}(s) \Delta s$ for sufficiently large $t_{2} \in\left(t_{1}, \infty\right)_{\mathbb{T}}$ for some $t_{1} \in\left[t_{0}, \infty\right)_{\mathbb{T}}$, is oscillatory, then the conclusion of Lemma 3.4 holds.

LEMMA 3.6. Let the condition (1.3) hold and assume that $x(t)$ is an eventually positive solution of the equation (1.2) and the corresponding y satisfies Case (II) of Lemma 3.1. If (2.22) holds, then $\lim _{t \rightarrow \infty} x(t)=0$.

LEMMA 3.7. Let $\hat{\zeta}_{1}$ be a nondecreasing on $\left[t_{0}, \infty\right)_{\mathbb{T}}$, the condition (1.3) hold and assume that $x(t)$ is an eventually positive solution of the equation (1.2) and the corresponding y satisfies Case (II) of Lemma 3.1. If

$$
\limsup _{t \rightarrow \infty} \int_{\hat{\zeta}_{1}(t)}^{t}\left(\frac{1}{a(u)} \int_{u}^{t} Q(s)\left[\hat{\zeta}_{1}(t)-\hat{\zeta}_{1}(s)\right]^{\lambda / \beta} \Delta s\right)^{1 / \alpha} \Delta u> \begin{cases}1 & \text { if } \lambda=\alpha \beta \\ 0 & \text { if } \lambda<\alpha \beta\end{cases}
$$

then $\lim _{t \rightarrow \infty} x(t)=0$.

LEMMA 3.8. Let the hypotheses of Lemma 3.7 hold with the condition (3.16) be replaced by

$$
\limsup _{t \rightarrow \infty} \int_{\hat{\zeta}_{1}(t)}^{t} \int_{v}^{t}\left(\frac{1}{a(u)} \int_{u}^{t} Q(s) \Delta s\right)^{1 / \alpha} \Delta u \Delta v> \begin{cases}1 & \text { if } \lambda=\alpha \beta, \\ 0 & \text { if } \lambda<\alpha \beta .\end{cases}
$$

Then the conclusion of Lemma 3.7 holds.

In the following two lemmas, we consider the second order delay dynamic equation

$$
\left(a(t)\left(w^{\Delta}(t)\right)^{\alpha}\right)^{\Delta}=\bar{d}\left(\bar{\zeta}_{2}(t)\right)^{\lambda / \gamma} \hat{Q}(t) w^{\lambda / \gamma}\left(\bar{\zeta}_{2}(t)\right),
$$

where $\bar{\zeta}_{2}$ and $\hat{Q}$ are as in (3.8) and (3.12) respectively, $a, \alpha, \gamma, \lambda$ are as in the equation (1.1) and $\bar{d}>0$ is a constant.

LEMMA 3.9. Let $\bar{\zeta}_{2}$ be a nondecreasing on $\left[t_{0}, \infty\right)_{\mathbb{T}}$ and the condition (1.3) hold. If

$$
\limsup _{t \rightarrow \infty} \int_{\bar{\zeta}_{2}(t)}^{t}\left(\bar{\zeta}_{2}(s)\right)^{\lambda / \gamma} \hat{Q}(s)\left(\int_{\bar{\zeta}_{2}(s)}^{\bar{\zeta}_{2}(t)} a^{-1 / \alpha}(\tau) \Delta \tau\right)^{\lambda \gamma} \Delta s> \begin{cases}\frac{1}{d} & \text { if } \lambda=\alpha \gamma, \\ 0 & \text { if } \lambda<\alpha \gamma,\end{cases}
$$

then all bounded solutions of the equation (3.18) are oscillatory. 


\section{THIRD ORDER NONLINEAR OSCILLATION}

P r o of. Let $w(t)$ be a bounded nonoscillatory solution of the equation (3.18), say $w(t)>0$ for $t \geq t_{1}$ for some $t_{1} \geq t_{0} \in \mathbb{T}$. Then there exists $t_{2} \in\left[t_{1}, \infty\right)_{\mathbb{T}}$ such that

$$
w(t)>0, w^{\Delta}(t)<0 \text { and } \quad\left(a(t)\left(w^{\Delta}(t)\right)^{\alpha}\right)^{\Delta}>0 \quad \text { for } t \geq t_{2} .
$$

Now for $v \geq u \geq t_{2}$, we have

$$
\begin{aligned}
w(u) & \geq w(u)-w(v)=-\int_{u}^{v} w^{\Delta}(\tau) \Delta \tau=-\int_{u}^{v} a^{-1 / \alpha}(\tau)\left(a(\tau)\left(w^{\Delta}(\tau)\right)^{\alpha}\right)^{1 / \alpha} \Delta \tau \\
& \geq-\left(a(v)\left(w^{\Delta}(v)\right)^{\alpha}\right)^{1 / \alpha} \int_{u}^{v} a^{-1 / \alpha}(\tau) \Delta \tau
\end{aligned}
$$

For $t \geq s \geq t_{2}$, setting $u=\bar{\zeta}_{2}(s)$ and $v=\bar{\zeta}_{2}(t)$ in the inequality (3.21) gives

$$
w\left(\bar{\zeta}_{2}(s)\right) \geq-\left(a\left(\bar{\zeta}_{2}(t)\right)\left(w^{\Delta}\left(\bar{\zeta}_{2}(t)\right)\right)^{\alpha}\right)^{1 / \alpha} \int_{\bar{\zeta}_{2}(s)}^{\bar{\zeta}_{2}(t)} a^{-1 / \alpha}(\tau) \Delta \tau .
$$

Integrating the equation (3.18) from $\bar{\zeta}_{2}(t) \geq t_{2}$ to $t$, we obtain

$$
\begin{aligned}
-a\left(\bar{\zeta}_{2}(t)\right)\left(w^{\Delta}\left(\bar{\zeta}_{2}(t)\right)\right)^{\alpha} & \geq a(t)\left(w^{\Delta}(t)\right)^{\alpha}-a\left(\bar{\zeta}_{2}(t)\right)\left(w^{\Delta}\left(\bar{\zeta}_{2}(t)\right)\right)^{\alpha} \\
& =\int_{\bar{\zeta}_{2}(t)}^{t} \bar{d}\left(\bar{\zeta}_{2}(s)\right)^{\lambda / \gamma} \hat{Q}(s) w^{\lambda / \gamma}\left(\bar{\zeta}_{2}(s)\right) \Delta s .
\end{aligned}
$$

Using (3.22) in (3.23), one can easily see that

$$
\begin{aligned}
& -a\left(\bar{\zeta}_{2}(t)\right)\left(w^{\Delta}\left(\bar{\zeta}_{2}(t)\right)\right)^{\alpha} \\
& \quad \geq\left(-a\left(\bar{\zeta}_{2}(t)\right)\left(w^{\Delta}\left(\bar{\zeta}_{2}(t)\right)\right)^{\alpha}\right)^{\frac{\lambda}{\alpha \gamma}} \int_{\bar{\zeta}_{2}(t)}^{t} \bar{d}\left(\bar{\zeta}_{2}(s)\right)^{\lambda / \gamma} \hat{Q}(s)\left(\int_{\bar{\zeta}_{2}(s)}^{\bar{\zeta}_{2}(t)} a^{-1 / \alpha}(\tau) \Delta \tau\right)^{\lambda / \gamma} \Delta s,
\end{aligned}
$$

or

$$
\left[-a\left(\bar{\zeta}_{2}(t)\right)\left(w^{\Delta}\left(\bar{\zeta}_{2}(t)\right)\right)^{\alpha}\right]^{1-\frac{\lambda}{\alpha \gamma}} \geq \int_{\bar{\zeta}_{2}(t)}^{t} \bar{d}\left(\bar{\zeta}_{2}(s)\right)^{\lambda / \gamma} \hat{Q}(s)\left(\int_{\bar{\zeta}_{2}(s)}^{\bar{\zeta}_{2}(t)} a^{-1 / \alpha}(\tau) \Delta \tau\right)^{\lambda / \gamma} \Delta s .
$$

Now take the limsup as $t \rightarrow \infty$ of both sides of the above inequality. If $\lambda=\alpha \gamma$ the contradiction is obvious. If $\lambda<\alpha \gamma$ the left hand side of (3.24) is positive and must decrease to zero (to present a contradiction to the positivity of $w(t)$ ). This contradicts (3.19) and completes the proof of the lemma. 
LEMMA 3.10. Let $\bar{\zeta}_{2}$ be a nondecreasing on $\left[t_{0}, \infty\right)_{\mathbb{T}}$ and the condition (3.19) in Lemma 3.9 be replaced by

$$
\limsup _{t \rightarrow \infty} \int_{\bar{\zeta}_{2}(t)}^{t}\left(\frac{1}{a(s)} \int_{s}^{t}\left(\bar{\zeta}_{2}(\tau)\right)^{\lambda / \gamma} \hat{Q}(\tau) \Delta \tau\right) \Delta s> \begin{cases}\bar{d}^{-1 / \alpha} & \text { if } \lambda=\alpha \gamma \\ 0 & \text { if } \lambda<\alpha \gamma\end{cases}
$$

Then the conclusion of Lemma $\mathbf{3 . 9}$ holds.

P r o of. Let $w(t)$ be a bounded nonoscillatory solution of the equation (3.18), say $w(t)>0$ for $t \geq t_{1} \geq t_{0} \in \mathbb{T}$. As in the proof of Lemma [3.9, we obtain (3.20) for $t \geq t_{2}$ for some $t_{2} \in\left[t_{1}, \infty\right)_{\mathbb{T}}$. Integrating (3.18) from $u \geq t_{2}$ to $t \geq u$, we have

$$
a(t)\left(w^{\Delta}(t)\right)^{\alpha}-a(u)\left(w^{\Delta}(u)\right)^{\alpha}=\int_{u}^{t} \bar{d}\left(\bar{\zeta}_{2}(s)\right)^{\lambda / \gamma} \hat{Q}(s) w^{\lambda / \gamma}\left(\bar{\zeta}_{2}(s)\right) \Delta s
$$

or

$$
-w^{\Delta}(u) \geq w^{\frac{\lambda}{\alpha \gamma}}\left(\bar{\zeta}_{2}(t)\right)\left(\frac{1}{a(u)} \int_{u}^{t} \bar{d}\left(\bar{\zeta}_{2}(s)\right)^{\lambda / \gamma} \hat{Q}(s) \Delta s\right)^{1 / \alpha} .
$$

Integrating this inequality from $\bar{\zeta}_{2}(t)$ to $t$, we obtain

$$
w\left(\bar{\zeta}_{2}(t)\right) \geq w^{\frac{\lambda}{\alpha \gamma}}\left(\bar{\zeta}_{2}(t)\right) \int_{\bar{\zeta}_{2}(t)}^{t}\left(\frac{1}{a(u)} \int_{u}^{t} \bar{d}\left(\bar{\zeta}_{2}(s)\right)^{\lambda / \gamma} \hat{Q}(s) \Delta s\right)^{1 / \alpha} \Delta u,
$$

or

$$
w^{\left(1-\frac{\lambda}{\alpha \gamma}\right)}\left(\bar{\zeta}_{2}(t)\right) \geq \int_{\bar{\zeta}_{2}(t)}^{t}\left(\frac{1}{a(u)} \int_{u}^{t} \bar{d}\left(\bar{\zeta}_{2}(s)\right)^{\lambda / \gamma} \hat{Q}(s) \Delta s\right)^{1 / \alpha} \Delta u .
$$

Taking the limsup as $t \rightarrow \infty$ of both sides of the above inequality, we again obtain a contradiction as in the previous lemma.

We are now ready to establish the main results of this section.

ThEOREM 3.1. Let $\lambda \leq \alpha \gamma, \lambda \leq \alpha \beta, \hat{\zeta}_{1}$ and $\bar{\zeta}_{2}, i=1,2$ be nondecreasing on $\left[t_{0}, \infty\right)_{\mathbb{T}}$ and the condition (1.3) hold. If either one of the dynamic equations (3.13), (3.14) or (3.15) is oscillatory, the condition (3.16) or (the condition (3.17)) holds and the condition (3.19) or (the condition (3.25) ) is satisfied with $0<\bar{d}<1$, then every solution $x(t)$ of the equation (1.2) oscillates or satisfies $\lim _{t \rightarrow \infty} x(t)=0$.

Proof. Let $x(t)$ be an eventually positive solution of the equation (1.2), say $x(t)>0$ and $x\left(h_{i}(t)\right)>0, i=1,2$ and $x(g(t, \tau))>0$ for $t \geq t_{1}$ 


\section{THIRD ORDER NONLINEAR OSCILLATION}

for some $t_{1} \in\left[t_{0}, \infty\right)_{\mathbb{T}}$ and $\tau \in[a, b]$. Then $y(t)$ defined by (3.1) satisfies one of the three cases of Lemma 3.1 and satisfies the equation (3.2). If Case (I) holds, then (3.3) holds. Now using (3.3) in the equation (3.2), we obtain the inequality (3.9). By either Lemma 3.2, Lemma 3.4 or Lemma 3.5. Case (I) cannot hold. Similarly, when Case (II) one can easily obtain the inequality (3.10), and by Lemma 3.7 or Lemma 3.8. Case (II) cannot hold. Finally, if Case (III) holds, we let $0<z(t)=-y(t)$ and proceed as above to obtain the inequality (3.11). It is easy to check that $z$ satisfies

$$
z(t)>0, \quad z^{\Delta}(t)>0, \quad z^{\Delta \Delta}(t)<0 \quad \text { and } \quad\left(a(t)\left(z^{\Delta \Delta}(t)\right)^{\alpha}\right)^{\Delta} \geq 0,
$$

for $t \geq t_{2} \geq t_{1}$. Now, there exists a constant $\bar{d} \in(0,1)$ such that

$$
z\left(\bar{\zeta}_{2}(t)\right) \geq \bar{d} \bar{\zeta}_{2}(t) z^{\Delta}\left(\bar{\zeta}_{2}(t)\right) \quad \text { for } \quad t \geq t_{3} \geq t_{2} .
$$

Using (3.26) in (3.11), we get

$$
\left(a(t)\left(z^{\Delta \Delta}(t)\right)^{\alpha}\right)^{\Delta} \geq \hat{Q}(t)\left(\bar{d} \bar{\zeta}_{2}(t) z^{\Delta}\left(\bar{\zeta}_{2}(t)\right)\right)^{\lambda / \gamma} \quad \text { for } \quad t \geq t_{3},
$$

or

$$
\left(a(t)\left(w^{\Delta}(t)\right)^{\alpha}\right)^{\Delta} \geq(\bar{d})^{\lambda / \gamma}\left(\bar{\zeta}_{2}(t)\right)^{\lambda / \gamma} \hat{Q}(t) w^{\lambda / \gamma}\left(\bar{\zeta}_{2}(t)\right) \quad \text { for } \quad t \geq t_{3},
$$

where $w(t):=z^{\Delta}(t)$. Proceeding as in the proofs of Lemma 3.9 and Lemma 3.10. we arrive at the desired conclusion completing the proof of the theorem.

Remark 3. We may note that Theorem 2.1 is also applicable to the equation (1.2). The details are omitted.

When $p(t, \theta) \equiv 0$ in the equation (1.1) or $\beta=1, r(t)=0$ and $h_{1}(t)=t$ in the equation (1.2), both equations are reduced to

$$
\left(a(t)\left(x^{\Delta \Delta}(t)\right)^{\alpha}\right)^{\Delta}+\int_{a}^{b} q(t, \tau) x^{\lambda}(g(t, \tau)) \Delta \tau=0 .
$$

In this case we have the following new result.

TheOREM 3.2. Let $\lambda \leq \alpha$ and (1.3) hold. If either one of the equations (2.7) with $p^{*} \equiv 0$, (2.10) or (2.17) with $p^{*} \equiv 0$ is oscillatory and the condition (2.27) or (the condition (2.31) holds, then every solution $x(t)$ of the equation (3.27) oscillates or satisfies $\lim _{t \rightarrow \infty} x(t)=0$.

Pr o o f. The conclusion follows from Theorem 2.2 or 3.1 and is omitted. 


\section{TAHER S. HASSAN — SAID R. GRACE}

\section{General remarks}

(1) The results of this paper are presented in a form that is essentially new and of a high degree of generality.

(2) We note that there are many criteria in the literature of first and second order dynamic equations and so by applying these results to the equations (2.7), (2.10) and (2.17), we can obtain many oscillation results, more that those known in the literature. Here we omit the details.

(3) The results here are valid for various type of time scales, e.g., $\mathbb{T}=\mathbb{R}$, $\mathbb{T}=\mathbb{Z}, \mathbb{T}=h \mathbb{Z}$ with $h>0, \mathbb{T}=q^{\mathbb{N}_{0}}$ with $q>1, \mathbb{T}=\mathbb{N}_{0}^{2}$, etc. (see [2]).

(4) We note that our results on the asymptotic behavior of solutions are applicable to the equations (1.1) and (1.2) if $g(t, \tau) \leq t, \tau \in[a, b]$ while our oscillation results are applicable to the equations (1.1) and (1.2) if $g(t, \tau)<t, \tau \in[a, b]$. Thus as it has been known, there is the delay in the equations (1.1) and (1.2) which can generate oscillations.

(5) Our results of Section 2 are new and our results of Section 3 include, extend and improve the results in [9] and [12].

(6) Finally, it would be of interest to consider the equations (1.1) and (1.2) try to obtain some oscillation criteria if other appropriate conditions on the functions $p(t, \theta), \theta \in[c, d], r(t)$, etc.

\section{REFERENCES}

[1] BOHNER, M.: Some oscillation criteria for first order delay dynamic equations, Far East J. Appl. Math. 18 (2005), 289-304.

[2] BOHNER, M.-PETERSON, A.: Dynamic Equations on Time Scales: An Introduction with Applications. Birkhäuser, Boston, 2001.

[3] DURINA, J.-BACULIKOVA, B.: Property (A) of third-order advanced differential equations, Math. Slovaca 64 (2014), 339-346.

[4] GRACE, S. R.-AGARWAL, R. P.-AKTAS, M. F.: On the oscillation of third order functional differential equations, Indian J. Pure Appl. Math. 39 (2008), 491-509.

[5] GRACE, S. R.-AGARWAL, R. P.-PAVANI, R.-THANDAPANI, E.: On the oscillation of certain third order nonlinear functional differential equations, Appl. Math. Comput. 202 (2008), 102-112.

[6] GRACE, S. R.-AGARWAL, R. P.-BOHNER, M.-O'REGAN, D.: Oscillation of second-order strongly superlinear and strongly sublinear dynamic equations, Commun. Nonlinear Sci. Numer. Simul. 14 (2009), 3463-3471.

[7] GRACE, S. R.-BOHNER, M.-AGARWAL, R. P.: On the oscillation of second-order half-linear dynamic equations, J. Difference Equ. Appl. 15 (2009), 451-460.

[8] GRACE, S. R.-R. P. AGARWAL, R. P.-KAYMAKALAN, B.-SAE-JIE, W.: On the oscillation of certain second order nonlinear dynamic equations, Math. Comput. Modelling 50 (2009), 273-286. 


\section{THIRD ORDER NONLINEAR OSCILLATION}

[9] GRACE, S. R.-GRAEF, J. R.-EL-BELTAGY, M. A.: On the oscillation of third order neutral delay dynamic equations on time scales, Comput. Math. Appl. 63 (2012), 775-782.

[10] GRAEF, J. R.-PANIGRAHI, S.-REDDY, P. R.: On oscillatory and asymptotic behavior of fourth order nonlinear neutral delay dynamic equations with positive and negative coefficients, Math. Slovaca 64 (2014), 347-366.

[11] HALE, J. K.: Theory of Functional Differential Equations. Springer-Verlag, New York, 1977.

[12] HAN, Z.-LI, T.-SUN, T.-ZHANG, C.: Oscillation behavior of third-order neutral Emden-Fowler delay dynamic equations on time scales, Adv. Difference Equ. 2010, Art. ID 586312, $23 \mathrm{pp}$.

[13] ELABBASY, E. M.-HASSAN, T. S.: Oscillation of solutions for third order functional dynamic equations, Electron. J. Differential Equations 2010, No. 131, 14 pp.

[14] ERBE, L.-PETERSON, A.-SAKER, S. H.: Hille and Nehari type criteria for third order dynamic equations, J. Math. Anal. Appl. 329 (2007), 112-131.

[15] ERBE, L.-HASSAN, T. S.-PETERSON, A.: Oscillation of third order functional dynamic equations with mixed arguments on time scales, J. Appl. Math. Comput. 34 (2010), 353-371.

[16] HASSAN, T. S.: Oscillation criteria for half-linear dynamic equations on time scales, J. Math. Anal. Appl. 345 (2008), 176-185.

[17] HASSAN, T. S.: Oscillation of third order nonlinear delay dynamic equations on time scales, Math. Comput. Modelling 49 (2009), 1573-1586.

[18] SAKER, S. H.-GRACE, S. R.: Oscillation criteria for quasi-linear functional dynamic equations on time scales, Math. Slovaca 62 (2012), 501-524.

Received September 6, 2014

Taher S. Hassan

Department of Mathematics

Faculty of Science

University of Hail

Hail, 2440

SAUDI ARABIA

Department of Mathematics

Faculty of Science

Mansoura University

Mansoura, 35516

EGYPT

E-mail: tshassan@mans.edu.eg

Said R. Grace

Department of Engineering Mathematics

Faculty of Engineering

Cairo University

Orman, Giza 12221

EGYPT

E-mail: srgrace@eng.cu.edu.eg 